\title{
COMBINING DIFFERENT SUBJECTS FROM AN INDUSTRIAL CHEMICAL ENGINEERING BACHELOR'S DEGREE FOR ADVANCED KNOWLEDGE ACQUISITION
}

\author{
Junkal Gutierrez, Zuriñe Gómez de Balugera, Josune Amurrio, Arrate Santaolalla, \\ \& Gorka Gallastegui \\ Department of Chemical and Environmental Engineering, Faculty of Engineering Vitoria-Gasteiz, \\ University of the Basque Country (Spain)
}

\begin{abstract}
This study intended to achieve advanced knowledge acquisition by creating effective linkages between specialised engineering subjects. Herein it is describing a pilot experience based on a problem-based methodology (PBM) involving third-year students taking the Industrial Chemical Engineering Bachelor's Degree at the Faculty of Engineering Vitoria-Gasteiz (University of the Basque Country (UPV/EHU)). In this case, students had to design lab reports regarding how to determine different target substances in real matrices, such as deodorant bottles, brass alloys or lab residues.

Despite the difficulties encountered by students as regards attaining the established objective (elaborate a lab script), the proposed activity was found to be an adequate tool for linking Analytical Chemistry and Chemical Engineering Laboratory II subjects, as well as an efficient method for internalizing theoretical knowledge. Performance of the activity was evaluated by means of a student survey and SWOT analysis. This experience could be transferred to other subjects related to Engineering or Applied Sciences.
\end{abstract}

Keywords: Active learning pedagogy, chemical education, chemical engineering degree, laboratory experiments, problem-based learning.

\section{Introduction}

Implementation of the European Higher Education Area following the Bologna Declaration resulted in a transformation of Industrial Engineering studies in Spain. The teaching plan for Industrial Engineering specialties (240 ECTS) (e.g., Mechanical Engineering, Industrial Electronic Engineering, and Industrial Chemical Engineering) is currently designed to ensure that students take the same courses for the first two years, with more specialised subjects being introduced throughout the third academic year, thus reducing the specific workload to 60 ECTS (Feijoo et al., 2018). This all adds up to a vast amount of theoretical knowledge that must be acquired over a short space of time.

In the case of Industrial Chemical Engineering, a second problem resulting from the reorganization of Bachelor studies promoted by the Spanish Ministry of Science and Innovation includes a reduction in laboratory hours. Indeed, several subjects, such as Analytical Chemistry, Physical Chemistry or Industrial Chemistry, have not planned to dedicate any time to laboratory practicals. As such, laboratory and practical work related to chemical engineering is exclusively taught in two sequential subjects (Experimentation in Chemical Engineering $I$ and $I I$ ) worth 6 ECTS credits each. This leads to the paradox that students carry out practical work in chemical engineering laboratories without having the prior knowledge needed to understand the tasks at hand. This is primarily the case because students have not yet undertaken the theoretical subjects directly related to this specialization. Figure 1 shows the educational pathway diagram proposed by the Chemical Engineering and Environmental Engineering Department of the Faculty of Engineering Vitoria-Gasteiz for correct adherence to the academic program for the Bachelor's Degree in Industrial Chemical Engineering.

Despite having access to this information, experience has shown that very few students follow the directions given above, performing laboratory practicals without having consolidated the theoretical content taught in subjects such as Unit Operations, Chemical Reaction Engineering or Analytical Chemistry.

In any case, even those students who following the suggestions in this figure to the letter have trouble understanding the practicals carried out in chemical engineering laboratories due to the limited time available to assimilate content given that theoretical subjects and practicals overlap throughout the academic year. 
Figure 1. Recommendations for correct adherence to the academic program.

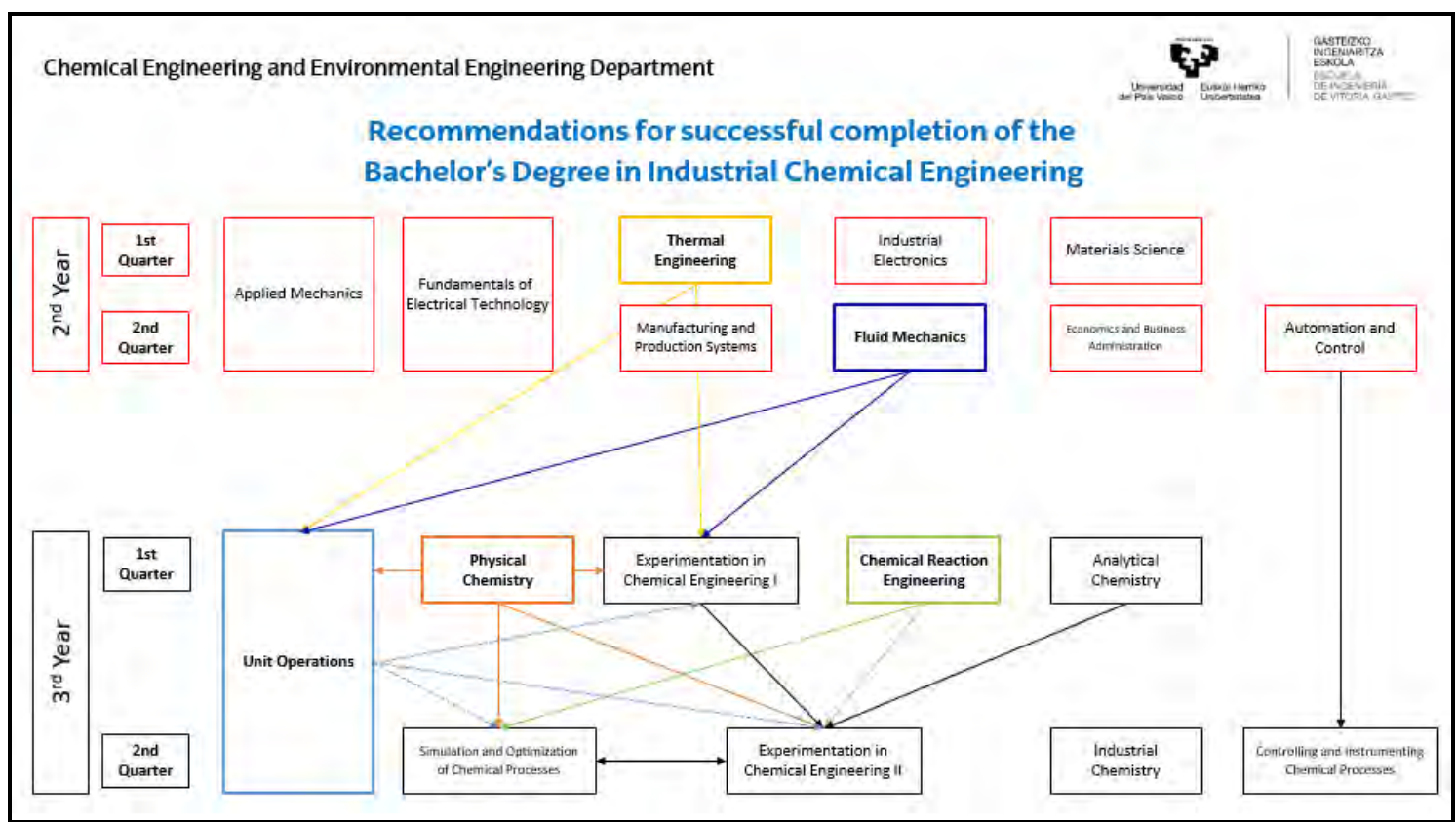

With a view to solving the aforementioned problems, this work aimed to achieve greater knowledge acquisition by creating effective linkages between specialised subjects. To that end, a joint project between Analytical Chemistry and Chemical Engineering Laboratory II was proposed.

\section{Framework}

This research project was applied during the 2018-2019 academic year with students studying for the Industrial Chemical Engineering Bachelor's Degree at the Faculty of Engineering Vitoria-Gasteiz (University of the Basque Country (UPV/EHU)). The interdisciplinary theoretical-practical framework comprised of two specialised subjects taught during the third academic year: Analytical Chemistry ( $1^{\text {st }}$ quarter) and Experimentation in Chemical Engineering II ( $2^{\text {nd }}$ quarter $)$.

Analytical Chemistry (6 ECTS) is devoted to providing an understanding of both quantitative chemical analysis and instrumental analysis, while Experimentation in Chemical Engineering II (6 ECTS) is considered a fundamental subject for acquiring the practical skills that graduates with a Bachelor's Degree in Industrial Chemical Engineering should demonstrate on a daily basis in their profession.

\section{Objective}

The aim of this approach was to improve the process by which students internalize theoretical knowledge that is subsequently applied in solving real problems, in other words implementation of a problem-based methodology (PBM) according to the following steps: create a team, analyse the problem, research, visualise all the possible solutions, debate, test and, finally, provide a solution.

\section{Methodology and development}

Thus, in the first subject, students were divided into groups of about 5 and asked to propose an experimental design by means of different experimental methods for real problems suggested by teaching staff (the first four tasks detailed in the previous section). In the practical session, during the course of the second subject, students completed the research following the self-designed lab-script and verified the feasibility of their proposal (the latter two activities).

The following real cases were proposed:

- Case 1 - Quantitative determination of aluminium in deodorant brands: the primary substances contained in deodorant bottles that help to keep the body dry (i.e., aluminium zirconium trichlorohydrex GLY24 or aluminium chlorohydrate) are aluminium-based (Sedwick, Leal, Turner, \& Kanu, 2018), since this element is able to clog sweat ducts in the areas to which it is applied. However, this is not an element that resides naturally in the 
human body and, therefore, analytical methods should be developed to determine its content in over-the-content products, such as deodorants.

- Case 2 - Determination of the chemical composition of a brass sample: according to DIN 1718, brass includes all copper-zinc alloys with more than $50 \%$ copper (wt \%). Additionally, it can contain up to $4 \%$ lead (Cintas Metálicas S.A. [CIMSA], (n.d.)). Due to its bright yellow colour, brass strongly resembles gold, therefore it is widely used in costume jewellery and decorative elements (Coca Rebolledo \& Rosique Jiménez, 2000). Depending on the amount of zinc in the alloy, other applications range from weaponry to boilermaking and welding. As such, analytical methods should be developed to determine the zinc content in brass alloys.

Going back to the problem previously posed in the introduction section (i.e., subject enrolment in the wrong order), a real-life case (Case 3) was designed for students undertaking Experimentation in Chemical Engineering II but not Analytical Chemistry. These students used a silver nitrate waste solution generated during laboratory practicals in Chemical Principles of Engineering (compulsory first-year subject) and were asked to plan and test a treatment to recycle this waste back into a new reagent, thereby reducing costs for the University and burdens on the environment.

The first two cases were presented at the beginning of the Analytical Chemistry course, and each student group was required to complete the following work assignments throughout the sessions:

1. Analyse the problem: a comprehensive understanding of the properties of the product and its applications should be achieved, including the theoretical amount of the target compound that is usually found within the matrix, as well as the influence of the latter.

2. Research: collect all the possible quantitative analysis techniques for determination of the target substances.

3. Visualise all the possible solutions: it should be kept in mind that proposed solutions have to comply with current legislation, taking into account the maximum permitted concentrations for these substances, etc.

4. Debate amongst themselves and with the supervisor: the best proposal should also take into consideration a number of factors, such as material resources, budget for the purchase of chemical reagents and time available, etc. Thus, the best available technique for practical implementation of the analysis and determination of the test sample will be selected from all the project proposals submitted.

Each of these issues led to a technical report that students presented in order to assess their work. This document had to include a laboratory manual comprising the following sections:

- Background and context

- Objective

- Theoretical foundation
- Experimental procedure (Materials and methods)

- Expected results

- References

For the second project phase (practical sessions), participants were required to:

5. Test and Confirm: the protocol proposed in the technical report was carried out in the chemical engineering laboratory (Figure 2).

6. Provide a solution: the necessary modifications were implemented, taking into account the results obtained in the previous section, to provide a satisfactory answer to the problem.

Figure 2. Example of student working on their self-designed lab-script and verifying the feasibility of their proposal.

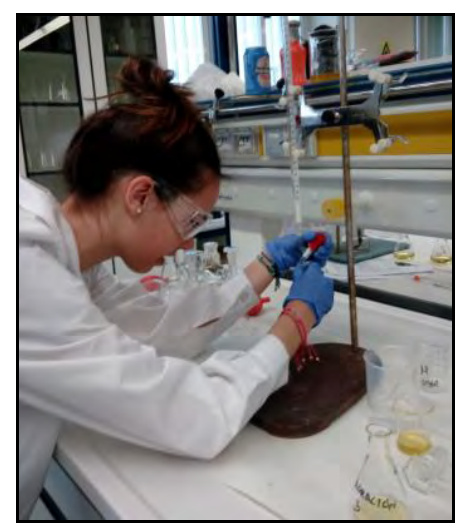

\section{Evaluation and informal feedback}

Project evaluation in terms of effective resolution of the three challenges posed was unsatisfactory given that students were unable to propose realistic experimental solutions to these problems or to draw up a draft protocol similar to an international standard. In our case, the origin of this 
problem is probably mostly related to the fact that our students had no previous experience with this kind of methodologies that enhance their social skills.

As students' survey results illustrated (Figure 3), this does not, however, mean that the pilot experience had not been very helpful: students were able to maintain a theoretical discussion of the possible experimental solutions, contrasting their own wrong approaches with the valid alternatives proposed by teaching staff. Indeed, it became obvious at the end of the study that students acquired a significant amount of basic theoretical knowledge related to analytical chemistry.

Figure 3. Comparison of survey results concerning quality and interest of the project, application of theoretical knowledge, student self-assessment and teacher evaluation.

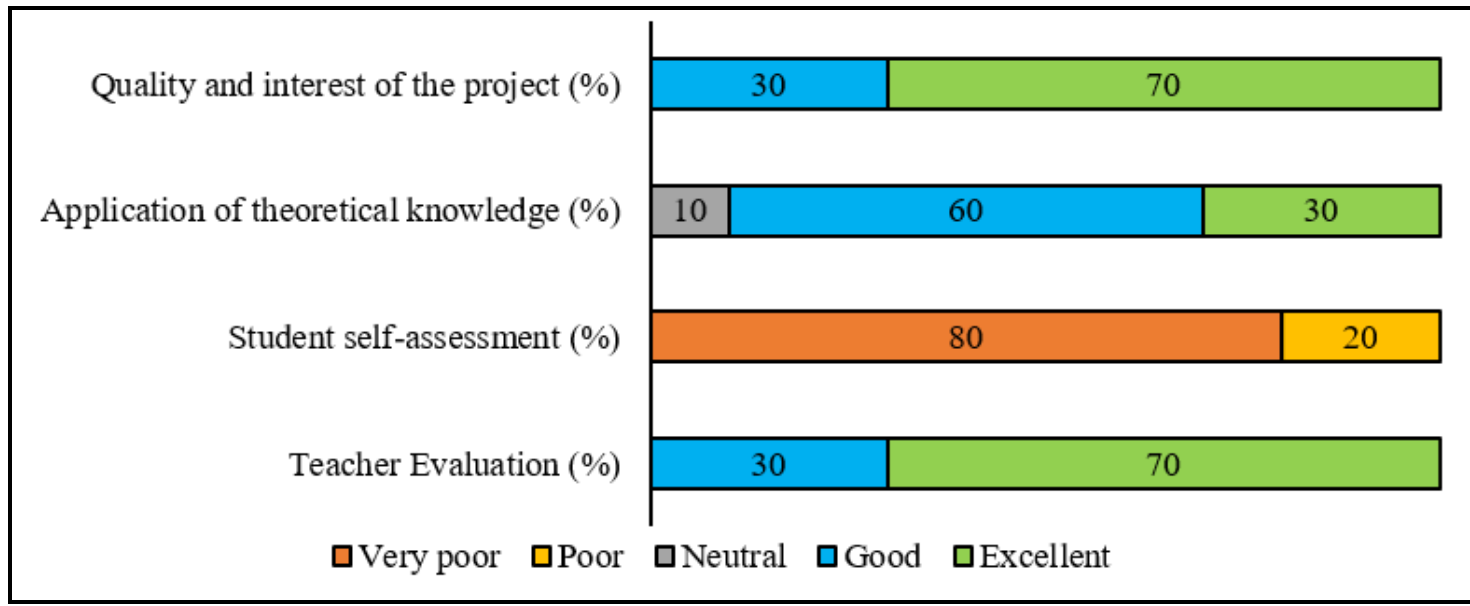

In addition, the survey showed that $70 \%$ of students considered this kind of activity to be an attractive way of learning. Given the value added to the learning program, they believed that similar projects should be carried out by establishing additional linkages between specialised subjects such as Chemical Reaction Engineering or Experimentation in Chemical Engineering II and Simulation and Optimization of Chemical Processes.

As regards student self-assessment, $80 \%$ of participants acknowledged a very poor misallocation of time, which finally resulted in poor-quality work at the end of the theoretical sessions. As far as teacher evaluation was concerned, $100 \%$ of participants considered that the approach adopted for the project, its monitoring, as well as coordination by teaching staff, was good or excellent.

\subsection{SWOT analysis}

After initial implementation of the PBM interdisciplinary study during the 2018-2019 academic year, both students and teachers carried out a careful reflection with a view to identifying areas of improvement. Table 1 shows a two-by-two grid that organizes the top strengths, weaknesses, opportunities, and threats by means of the SWOT analysis tool.

Table 1. SWOT analysis of the PBM example linking specialised subjects in the Industrial Chemical Engineering Bachelor's Degree.

\begin{tabular}{ll}
\hline \hline STRENGTHS & WEAKNESSES \\
\hline \hline $\begin{array}{l}\text { Students realize the applicability of theoretical } \\
\text { knowledge in real cases }\end{array}$ & $\begin{array}{l}\text { Experimentation in Chemical Engineering II and } \\
\text { Analytical Chemistry students are not the same }\end{array}$ \\
\hline $\begin{array}{l}\text { Close monitoring of learning achievements: teachers } \\
\text { receive detailed feedback regarding the theoretical } \\
\text { concepts understood by students }\end{array}$ & Non-compliance with the timescales \\
\hline Enhancement of critical analysis & $\begin{array}{l}\text { Lack of coordination within the group, which results in } \\
\text { inequitable distributions of the workload }\end{array}$ \\
\hline $\begin{array}{l}\text { Encourages communication ability and acquisition of } \\
\text { debating skills within the group and with supervisors }\end{array}$ & Weak self-demanding attitude \\
\hline \hline OPPORTUNITIES & THREATS \\
\hline \hline Possibility of extending the experience to other subjects & $\begin{array}{l}\text { Lack of time to complete the work if similar projects are } \\
\text { simultaneously developed }\end{array}$ \\
\hline $\begin{array}{l}\text { Ascertainment that engineering challenges have many } \\
\text { solutions }\end{array}$ & $\begin{array}{l}\text { The requirement to expose new challenges every } \\
\text { academic course to avoid any plagiarism }\end{array}$ \\
\hline
\end{tabular}




\section{Further improvements}

Based on this evidence, a series of improvement actions were proposed to minimize the weaknesses detected previously:

- The performance of assigned tasks in theoretical sessions should be followed-up in a more personalized, closer and speedier way in order to avoid leaving the toughest issues to the end.

- The role of group-coordinator should be established during the team-creation step, and periodic meetings should be held with this person in order to ensure the involvement of all group members in the project.

- The performance of the project in a staggered manner should be guaranteed by coordination with teaching staff and the setting of common rules.

\section{References}

CIMSA. (n.d.). Latones. Retrieved March 30, 2019, from http://www.cimsaww.com/internet/es/cupropedia/aleaciones_1/serie_m_zzerie_m_z.jsp.

Coca Rebollero, P., \& Rosique Jiménez, J. (2000). Ciencia de Materiales: Teoría - Ensayos- Tratamientos. Madrid: Ediciones Pirámide. ISBN 84-368-0404-X.

Feijoo, G., Ibañez, R., Herguido, J., Partal, P., Tobajas, M., Sèmpere, J., López-Pérez, M. F., \& Rivero, M. J. (2018). Education of chemical engineering in Spain: A global picture. Education for Chemical Engineers, 24, 27-31.

Sedwick, V., Leal, A., Turner, D., \& Kanu, A.B. (2018). Quantitative determination of aluminium in deodorant brands: a guided inquiry learning experience in quantitative analysis laboratory. Journal of Chemical Education, 95, 451-455. 